\title{
Electrically-Induced Causes Resistive Switching in Copper Helical Metallopolymers \\ Mixed Valence
}

Jake L. Greenfield ${ }^{1 \dagger}$, Daniele Di Nuzzo ${ }^{2 \dagger \star}$, Emrys W. Evans ${ }^{2}$, Satyaprasad P. Senanayak ${ }^{2}$, Sam Schott ${ }^{2}$, Jason T. Deacon ${ }^{1}$, Adele Peugeot ${ }^{1}$, William K. Myers ${ }^{3}$, Henning Sirringhaus ${ }^{2}$, Richard H. Friend ${ }^{2 *}$, Jonathan R. Nitschke ${ }^{1 *}$

\footnotetext{
Kingdom

† Contributed equally

* Corresponding authors
}

1 Department of Chemistry, University of Cambridge, Lensfield Road, Cambridge, CB2 1EW, United Kingdom

2 Cavendish Laboratory, University of Cambridge, JJ Thomson Avenue, Cambridge, CB3 OHE, United Kingdom

3 Centre for Advanced ESR, Department of Chemistry, University of Oxford, South Parks Road, Oxford, OX1 3QR, United

Correspondence to:

dd467@cantab.ac.uk

rhf10@cam.ac.uk

irn34@cam.ac.uk

\begin{abstract}
:
Controlling the flow of electrical current at the nanoscale typically requires complex top-down approaches. Here we use a bottom-up approach to demonstrate resistive switching within molecular wires that consist of double-helical metallopolymers and are constructed by self-assembly. When we expose the material to an electric field, we determine that approximately $25 \%$ of the copper atoms oxidise from $\mathrm{Cu}^{\prime}$ to $\mathrm{Cu}^{\prime \prime}$, without rupture of the polymer chain. The ability to sustain such high level of oxidation is unprecedented in a copper-based molecule: it is made possible here by the double helix compressing in order to satisfy the new coordination geometry required by $\mathrm{Cu}^{\prime \prime}$. This mixed-valence structure exhibits a $10^{4}$-fold increase in conductivity, which is projected to last over 10 years. We explain the increase in conductivity as being promoted by the creation, upon oxidation, of partly filled $d_{z}^{2}$ orbitals aligned along the mixed-valence copper array; the long-lasting nature of the change in conductivity is due to the structural rearrangement of the double-helix, which poses an energetic barrier to re-reduction. This work establishes helical metallopolymers as a new platform for controlling currents at the nanoscale.
\end{abstract}


Organic-based molecular materials offer the structural complexity necessary to build functionality at the nanoscale and from the bottom up. ${ }^{1-3}$ In hybrid metal-organic materials, the oxidation state of metal ions provides a further handle to engineer new properties ${ }^{4-11}$. Manipulation of the metal oxidation state in such systems impacts their electrical properties: switching of bulk resistivity in thin films has indeed been demonstrated via changing the oxidation state of the metal centres in metallopolymers ${ }^{7,9,10}$. In these reports, however, the metal ions are not organised into well-defined structures and are often separated from each other by organic moieties ${ }^{4-11}$. This results in limited metal-metal communication, and ultimately in poor nanoscale control of the metal system, due to the inherent disorder in polymer films.

An appealing approach to create ordered structures at the nanoscale is to place the metal centres in direct communication with each other. To this end, systems that contain linear chains of metal ions have been reported $d^{2,6,20-24,12-19}$. Of these, extended metal atom chains (EMACs) are molecules in which the metal ions in the chain are electronically coupled, permitting the development of novel nanoelectronic devices ${ }^{18,21-23}$. The oxidation state of the metal ions in these materials has a critical impact on the electrical conductivity, with mixedvalence along the array creating a delocalized electronic system ${ }^{16,22,23}$. While these previous works have investigated the impact of native mixed-valence on the electronic properties, however, the external control of the oxidation state remains to be explored, and exploited in functional devices.

Here we describe the electrical behaviour of a self-assembled, solution-processable doublehelical metallopolymer, which consists of a linear array of $\mathrm{Cu}^{\prime}$ ions coordinated to two organic polymeric ligand strands. Methods have been developed to control the regiochemistry, enantiomeric excess, and lengths of such polymers ${ }^{25,26}$. Upon exposure to an electric field, we find the polymer to adopt a novel mixed-valence structure, wherein approximately $25 \%$ of the copper atoms in the chain oxidise from $\mathrm{Cu}^{\prime}$ to $\mathrm{Cu}^{\prime \prime}$, without rupture of the polymer chain. Instead, the helically-wrapped ligand strands compress and wind more tightly about the $\mathrm{Cu}$ "l centres, adapting to the preferred geometry of the metal ion in this oxidation state and taking up radical-anion character to balance the increased charge on the metal core. This process results in an increase in conductivity of thin films by four orders of magnitude, and the more conductive state is extrapolated to last for several years at $300 \mathrm{~K}$. We conclude that the increase in conductivity is promoted by efficient charge transport via partly filled $d_{z}{ }^{2}$ orbitals extending along the mixed-valence copper array, and that the longlasting nature of the high-conductivity state is due to structural re-organisation of the polymer chain, which presents an energetic barrier to $\mathrm{Cu}^{\prime \prime}$ re-reduction. This linear metallopolymer is thus an EMAC which can be rendered conductive using an external electrical stimulus. Our work introduces copper double-helical metallopolymers as an easily processable and tailorable system, based on the earth-abundant metal copper, for the bottom-up creation and control of conductive atomic chains. 


\section{Results}

a<smiles>Nc1ncccc1C=O</smiles>

A

C





b



Fig. 1 | Self-assembly of metallopolymer and resistive switching. a, Subcomponent self-assembly of helical metallopolymer 1 from monomer A, end-cap B, and $\mathbf{C u}^{\prime}$. b. Schematic representation of $\mathbf{1}$, depicting a pair of conjugated polymer strands wrapped helically around a linear array of $\mathrm{Cu}^{\prime}$ centres. c, The device architecture employed to study charge transport through a $50 \mathrm{~nm}$ thickness of 1 . The inset depicts a schematic representation of 1 dispersed between the gold electrodes. d, Absolute value of current density vs. voltage for a 50mer of metallopolymer 1, showing the higher currents obtained as the device was scanned to progressively higher maximum voltages $\left(\mathrm{V}_{\max }\right)$. The arrows and numbers in the inset show how the voltage was modulated during each set of scans: first positive from zero, then back through zero and negative, then back to zero again. For the set where $V_{\max }=100 \mathrm{~V}$, the scan time between 0 and $V_{\max }$ was 115 seconds, for a total of 460 seconds for the four consecutive scans. For all the other curves it was 15 seconds, for a total of 60 seconds for the four scans. The same electrical behaviour was observed on glass substrates. The substrates were washed and re-used several times, showing no sign of damage to the $\mathrm{SiO}_{2}$ layer caused by operation.

Copper-based double-helical metallopolymer 1 was prepared by self-assembly of monomer A (100 equiv) with a small amount of end-cap B (2 equiv) and $\mathrm{CuNTf}_{2}$ (50.5 equiv; $\mathrm{Tf}=$ $\mathrm{O}_{2} \mathrm{SCF}_{3}$ ) in MeCN (Fig. 1a, b) ${ }^{25,26}$. We estimate the spacing between the Cu' centres within 1 to be $0.3 \mathrm{~nm}$ based upon the crystal structure of a corresponding oligomer ${ }^{25}$ and electron micrographs of the polymer in an aggregated state ${ }^{26}$. The $\mathbf{B}$ termini have been shown both to serve as chain-capping agents to control polymer length, and to dictate the handedness of the helical polymer strands ${ }^{26}$; in this study an A-to-B ratio was chosen to produce chains of $P$ handedness $(>52 \% \text { helical excess, measured using circular dichroism })^{26}$ that were an average of 50 repeat units long (measured using dynamic light scattering) ${ }^{26}$. Such oligomers were found to self-organise into tightly-packed intermolecular aggregates ${ }^{26}$, the formation of which is favoured by enantiomeric excess.

The co-existence of twin conjugated organic strands with a linear array of redox-active metal ions within 1 raises the question of which pathway would electrical charges follow in this polymer. To shed light on this question, we set out to investigate the electrical properties in 
the solid state. Thin films of 1 were prepared by spin coating, showing high aspect ratio rodlike structures tens of $\mathrm{nm}$ long and averaging $10 \mathrm{~nm}$ across, oriented preferentially in the plane of the substrate (Fig. S4) ${ }^{26}$. To investigate the electrical properties of 1 in the plane of the metallopolymer chains, we therefore constructed in-plane devices as shown in Fig. 1c, consisting of two gold electrodes deposited on silicon dioxide, separated by a distance of 10 $\mu \mathrm{m}$ and with electrode widths of $700 \mu \mathrm{m}$, onto which we deposited thin $(50 \mathrm{~nm})$ films of 1 .

We performed current-voltage scans (Fig. 1d) and observed a combination of transient and long-lasting effects, with the current density depending on the scan speed and on the maximum absolute voltage reached $\left(\mathrm{V}_{\max }\right)$. Taking each time a fresh device, four currentvoltage scans were performed, as noted in the inset of Fig. 1d. Scans (1) and (2) trace the forward and return scans, respectively, with positive voltage bias applied, and scans (3) and (4) represent the forward and return scans under negative voltage bias (Fig. 1d, inset). For $\mathrm{V}_{\max }$ values of 40 and $60 \mathrm{~V}$, both positive and negative bias scans showed substantial hysteresis, with the current densities in (2) and (4) significantly higher than in (1) and (3), respectively. For $\mathrm{V}_{\max }=80 \mathrm{~V}$, however, scans (3) and (4) showed negligible hysteresis and higher currents than the corresponding forward scans (1) and (2). When scans were performed at a slower rate $\left(0.6 \mathrm{~V} \mathrm{~s}^{-1}\right)$ reaching $\mathrm{V}_{\max }=100 \mathrm{~V}$, the current density was observed to reach the same values in (2) as in (3) and (4), as well as displaying a linear dependence on voltage above $50 \mathrm{~V}$ (Fig. S5). This linear dependence indicates that the device undergoes ohmic injection and transport above $50 \mathrm{~V}$ : under these conditions, a bulk conductivity of $2 \mathrm{~S} \mathrm{~m}^{-1}$ was estimated (Supplementary Information Section 4.1). Despite the high potentials employed, given the distance between the electrodes $(10 \mu \mathrm{m})$ the electric field applied across the metallopolymer films is moderate $\left(10^{7} \mathrm{~V} \mathrm{~m} \mathrm{~m}^{-1}\right.$ at $100 \mathrm{~V}$, comparable to the field applied to an OLED under standard operating conditions).

These results are rationalized as follows. The initial effect of the external electric field is to drive ion motion, and the ${ }^{-} \mathrm{NTf}_{2}$ counteranions migrates to the positively charged electrode to generate surface-charge-dipole (SCD) regions at the electrodes. These regions can facilitate charge injection, as is observed in devices such as Light-Emitting Electrochemical Cells ${ }^{27}$. In the slower $100 \mathrm{~V}$ scan of Fig. 1d, the current density during sweep (1) deviates from the trend observed in the faster scans, indicating that ion migration has had time to take place and facilitate charge injection. The effects of ion migration are transient, causing device properties to return to their initial conditions when the voltage bias is switched off. When the applied voltage is sufficiently high, or at lower scan rates $(80 \mathrm{~V}$ and $100 \mathrm{~V}$ curves, Fig. 1d), an additional, long-lasting change in the material is observed, which causes the devices to enter a low resistivity state independent of the scan polarity, removing hysteresis and asymmetry between scans in Fig. 1d. This resistive switching effect is inferred to result from a structural change taking place in the bulk of the material, as described below. 


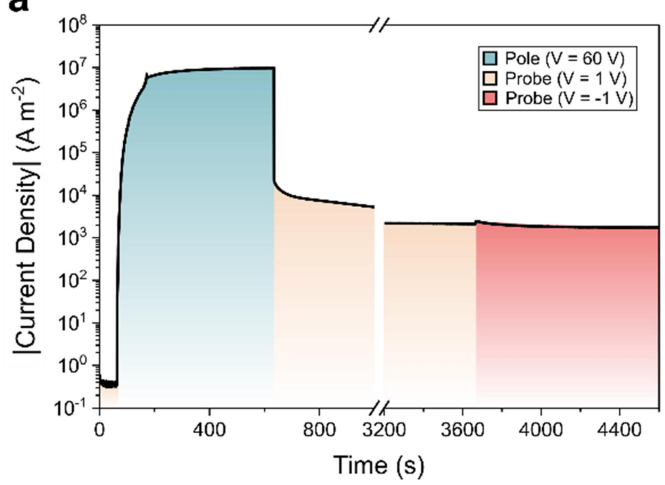

C

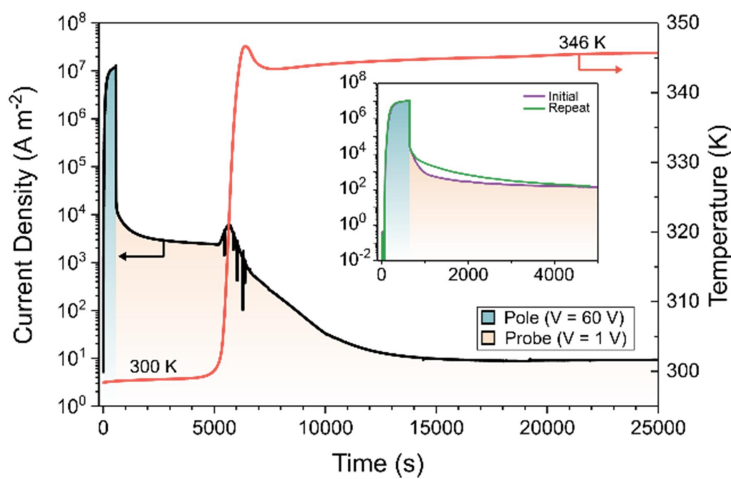

b

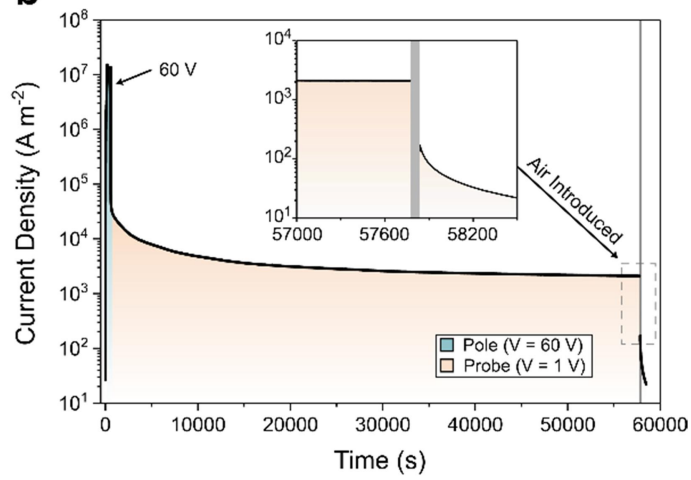

d

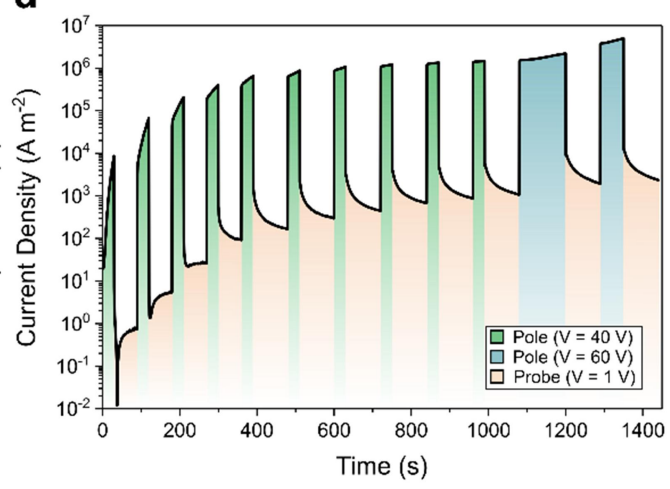

Fig. 2 | Resistive switching under different operating conditions. Current density as a function of time and voltage bias recorded on devices having the structure shown in Fig. 1c, containing metallopolymer 1 . Unless specified, the measurements were performed at constant temperature $(300 \mathrm{~K})$ and under vacuum $\left(10^{-5} \mathrm{Bar}\right)$. $\mathbf{a}$, Evolution following the application of subsequent "probe" ( $1 \mathrm{~V}$ or $-1 \mathrm{~V})$ and "pole" $(60 \mathrm{~V})$ voltage steps. The Y-axis shows the absolute current density, in order to display the results obtained at different voltage polarities. $\mathbf{b}$, Evolution on a longer time scale, with the device exposed to air after 16 hours biased at $1 \mathrm{~V}$. c, Effect of temperature on current density. The inset shows the reversibility of the effect: after heating the device to $346 \mathrm{~K}$, the current density recovered to the same value as was observed for the pristine material. $\mathbf{d}$, Sequential pole and probe cycles showing the different resistance states of the device accessed as a function of total pole duration; the current density value plateaued at ca. $900 \mathrm{~s}$ when using $40 \mathrm{~V}$ pole voltage and increased further following the application of a $60 \mathrm{~V}$ pole voltage.

To probe the resistive switching behaviour of these devices in more detail, we investigated the current density as a function of time using different applied voltages. The application of 1 $\mathrm{V}$ between the two gold contacts to a pristine device resulted in a current density of ca. $0.5 \mathrm{~A}$ $\mathrm{m}^{-2}$ (Fig. 2a). Increase of the potential to $60 \mathrm{~V}$ caused the current density to increase rapidly, reaching a plateau at ca. $10^{7} \mathrm{~A} \mathrm{~m}^{-2}$. Consistent with the results described above (Fig. $1 \mathrm{~d}$, slow scan, $V_{\max }= \pm 100 \mathrm{~V}$ ), this current density corresponds to a conductivity of $1.7 \mathrm{~S} \mathrm{~m}^{-1}$ (Supplementary Information Section 4.1). When the potential across the device was switched to $1 \mathrm{~V}$, the current density settled between $10^{3}$ and $10^{4} \mathrm{~A} \mathrm{~m}^{-2}$, four orders of magnitude higher than in the pristine state (i.e. the state before the $60 \mathrm{~V}$ 'pole' step was applied). Inverting the potential to $-1 \mathrm{~V}$ (after $1 \mathrm{~h}$ at $+1 \mathrm{~V}$ ) did not affect the current density, indicating that the conductance of the device is independent of the polarity of the 'probe' voltage. This polarity independence implies that the change in current density induced by electric poling is related to a change in the bulk conductivity of the material as opposed to a change in the injection properties. Note that in Fig. 2a the devices were poled for 570 seconds at a constant voltage bias of $60 \mathrm{~V}$. These conditions represent a significantly longer and stronger poling than in the short scans of Fig. 1d (60 seconds for the four consecutive 
scans between $+60 \mathrm{~V}$ and $-60 \mathrm{~V})$ : indeed, full and long-lasting resistive switching is observed in Fig. $1 \mathrm{~d}$ only when a slow scan is used (460 $\mathrm{s}$ in total, reaching $\pm 100 \mathrm{~V}$ ).

We observed the high-conductivity state obtained after poling at $60 \mathrm{~V}$ to remain with negligible loss for over 16 hours (Fig. 2b); we fitted the current density decay in the $1 \mathrm{~V}$ probe step to a power law $a / t^{b}+c$, where $c$ is fixed at $0.5 \mathrm{~A} \mathrm{~m}^{-2}$. Extrapolating this decay shows that under our experimental conditions $(1 \mathrm{~V}, 300 \mathrm{~K}$, in vacuum) the high conductivity state could in principle be retained for years. Indeed, if the observed decay was maintained indefinitely $\mathrm{y}^{28}$, it would take 12 years for the current density to decrease to $5 \mathrm{~A} \mathrm{~m}^{-2}$, i.e. a value 10 times higher than the initial value of $0.5 \mathrm{~A} \mathrm{~m}^{-2}$ recorded at $1 \mathrm{~V}$ before poling. The factor of 10 is chosen here as a safe threshold above which it would still be possible to read the written information, i.e. distinguish between a poled and a non-poled device by recording current density in presence of measurement noise; if a threshold factor of 2 is chosen, extrapolation indicates a retention time of 330 years (Fig. S7).

Exposing the poled device to air brought about a rapid decrease in current density (Fig. 2b). Measurements performed on devices that had been exposed to air while in the poled state showed a much slower build-up of current at $60 \mathrm{~V}$, reaching a plateau at 1000 times lower current density (Fig. S17). This air sensitivity was only exhibited by films that had been subjected to electric fields: the conductivity of virgin films of 1 was found to be independent of prior exposure to air. This observation indicates that poled 1 degrades upon exposure to air, implying that the resistive switching mechanism involves the generation and retention of air-sensitive species. Such degradation might involve disruption of the polymer chains, as supported by NMR studies (Supplementary Information Section 3.10).

By raising the temperature of a poled device, the low-conductivity state was recovered (Fig. $2 \mathrm{c}$ ): when a device that had been 'written' at $60 \mathrm{~V}$ for $9.5 \mathrm{~min}$ was heated to $346 \mathrm{~K}$ for $1.5 \mathrm{~h}$, under vacuum and no electrical bias, the initial low-conductivity state was restored. This recovery was reversible; subsequent operation of the thermally-reset device revealed no appreciable signs of sample degradation, reaching similar values of current density when repeating the full experiment (Fig. 2c, inset).

Finally, we performed short consecutive probe and pole cycles on our devices. Through applying a series of $30 \mathrm{~s}$ poling cycles at $40 \mathrm{~V}$, different conductivity states of the device were accessed (Fig. 2d). The number of distinct conductivity states depended upon the duration of the pole cycle: longer poling cycles brought the metallopolymer further towards its maximum conductivity at a given voltage. Subsequent operation at $60 \mathrm{~V}$ (Fig. 2d) further increased the number of accessible states.
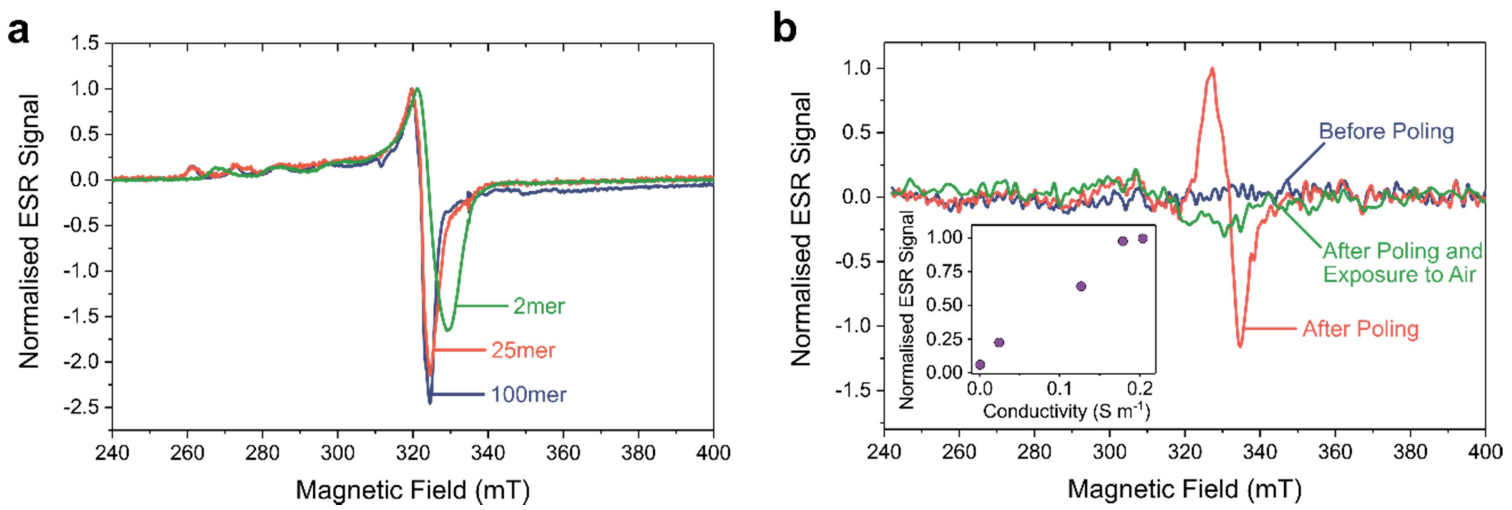

Fig. 3 | ESR evidence of $\mathrm{Cu}^{\prime \prime}$ formation upon electrical poling. a, Solution-state ESR measurements of oligomers of 1 with 2, 25, and 100 repeat units recorded in DMSO at $100 \mathrm{~K}$ under $\mathrm{N}_{2}$. b, In-situ ESR measurements of an in-plane device containing 50-repeat-unit metallopolymer 1 being poled under $\mathrm{N}_{2}$ at $300 \mathrm{~K}$. 
The device consisted of two gold electrodes deposited on quartz, separated by a distance of $100 \mu \mathrm{m}$ and with a width of $24.3 \mathrm{~mm}$, on top of which was deposited a $50 \mathrm{~nm}$ film of 1. The inset shows the normalised ESR signal as a function of conductivity recorded at the end of each poling step. Electrical characterisation and description of the experimental setup are provided in Supplementary Information Section 3.8.2.

We propose that the mechanism of resistive switching within 1 is related to a change in the oxidation states of the linear array of copper centres. Traces of $\mathrm{Cu}^{\prime \prime}$ in 1 that had not been poled were observed by XPS (Fig. S9) and by low-temperature ESR of 1 in frozen DMSO solution (Fig. 3a). In the frozen-solution spectra, the presence of a low-field quartet, paired with $g$-values ranging from $g_{\perp}=2.07-2.08$, was consistent with a population of $\mathrm{Cu}^{\prime \prime}(I=3 / 2)$ possessing axial symmetry and a $\mathrm{d}_{x-y}^{2}{ }^{2}$ SOMO (see Table $\mathrm{S} 1$ in Supplementary Information $)^{29,30}$.

In the pristine (unpoled) state of 1 , the location of the $\mathrm{Cu}^{\prime \prime}$ sites within the polymer core is thus fixed. This interpretation is supported by the observation of a low-field quartet (Fig. 3a), which implies hyperfine coupling of the unpaired electron to a single $\mathrm{Cu}$ nucleus ${ }^{29}$. Shorter oligomers were found to contain a higher proportion of $\mathrm{Cu}^{\prime \prime}$, ranging from ca. $21 \% \mathrm{Cu}^{\mathrm{II}}$ in a 2 mer to ca. $4 \%$ in a 100 mer (Fig. 3a and Table S1). These data suggested that, in the unpoled state, $\mathrm{Cu}$ " is spontaneously generated in small amounts. The inverse proportionality between the amount of $\mathrm{Cu}^{\prime \prime}$ and polymer length indicates that $\mathrm{Cu}^{\prime \prime}$ may reside at the termini of 1 , where greater ligand flexibility facilitates reconfiguration ${ }^{31}$ of the ends of the helix to satisfy the square-planar coordination geometry preference of $\mathrm{Cu}^{\text {Il }}{ }^{32}$. No inter-valence charge transfer (IVCT) bands were observed in the near-infrared spectral region of a pristine 50 mer of 1 , which would have indicated the migration of discrete $\mathrm{Cu}^{\text {II }}$ centres along the chain $^{33}$. We hypothesise that the charges of the $\mathrm{Cu}^{\text {Il }}$ sites at the termini of pristine 1 are balanced by radical anions hosted by the $\pi^{*}$ orbitals of the conjugated organic ligands in the ground state. Supporting this hypothesis, UV-vis absorption spectra of metallopolymer 1 displayed a low intensity absorption band at $950 \mathrm{~nm}$, consistent with the presence of quinoline-type radical anions, as was also observed in solution-state transient absorption measurements (Fig. S10) ${ }^{32}$.

We then employed in-situ ESR spectroscopy to study the effects of poling on the oxidation state of the copper chain of metallopolymer 1 at $300 \mathrm{~K}$. In-plane devices based on the structure shown in Fig. 1c, but with an inter-electrode separation of $100 \mu \mathrm{m}$, were suspended under nitrogen in an air-tight ESR tube, which allowed the device to be poled and its ESR spectrum recorded without altering its position in the microwave cavity (Supplementary Information, Section 3.8.2). The X-band ESR spectrum did not reveal any ESR signal for the device in the unpoled state (Fig. 3b), implying an absolute number of spins at room temperature below the detection limit of the instrument, estimated at $10^{13}$ spins: given the volume of sample and of the polymer repeat unit (see discussion below), this limit allows us to estimate that no more than ca. $0.5 \%$ of the Cu centres are in the $\mathrm{Cu}^{\prime \prime}$ state before poling at room temperature. We ascribe the lower density of $\mathrm{Cu}^{\prime \prime}$ in the in-situ device experiments than in the frozen solution described above to a more rigid configuration of the helix in the solid state ${ }^{26}$, as well as to the lower temperature at which the measurements were performed. Following poling at $20 \mathrm{~V}$ and removal of the electric field across the device, a clear ESR signal appeared between 320 and $350 \mathrm{mT}$. Successive $5 \mathrm{~min}$ / $20 \mathrm{~V}$ poling steps resulted in an increase of the maximum current density, and hence the conductivity, at the end of each step. The intensity of the ESR signal increased with the conductivity of the device (Fig. 3b, inset). All ESR signal intensity was lost upon exposing the device to air. 
We attribute the ESR signal observed in the poled devices to $\mathrm{Cu}^{\prime \prime}$ in the polymer chain. This assignment is in agreement with previous studies on $\mathrm{Cu}^{\text {Il }}$ arrays in artificial DNA ${ }^{2}$ and with the ESR spectrum of 1 in frozen solution (Fig. 3a). From the intensity of the ESR signal in the poled device (Fig. $3 b$ ) we estimate the presence of $(8 \pm 4) \times 10^{14} \mathrm{Cu}^{11}$ centres in the sample at maximum poling ( 5 minutes at $20 \mathrm{~V}$ repeated 5 times, reaching $0.2 \mathrm{~S} \mathrm{~m}^{-1}$ in these experiments, Fig. S14 and inset in Fig. 3b). Based on the active volume of 1 in the device and the volume of the repeat unit $\left(4.07 \times 10^{-28} \mathrm{~m}^{3}\right.$, determined from the crystal structure of a tetra-copper oligomeric analogue of 1$)^{25}$, we calculate that this value corresponds to $(27 \pm 13.5) \%$ of $\mathrm{Cu}^{\prime}$ atoms in 1 converting to $\mathrm{Cu}^{\prime \prime}$ with poling. In poled devices, the measured $g$-value is $g_{\perp}=2.03$, less than the value of 2.07-2.08 observed in frozen solutions and closer to the value for a free electron $\left(g_{\mathrm{e}}=2.00\right)$. This suggests the presence of $\mathrm{a} \mathrm{d}_{\mathrm{z}}^{2}$ based SOMO for $\mathrm{Cu}^{1134,35}$, where the $z$-axis coincides with the copper chain ${ }^{31}$. As explained in more detail below, this $\mathrm{d}_{\mathrm{z}}{ }^{2}$ SOMO configuration reflects Jahn-Teller distortion upon oxidation of $\mathrm{Cu}^{\prime}$ to $\mathrm{Cu}^{\prime \prime}$. We conclude that radicals on the organic strands of the metallopolymer were not the main contributors to the ESR signal because the measured $g_{\perp}$ deviated further from 2.00 than typical $g$-values of organic radicals, either free ${ }^{31,36}$ or bound within $\mathrm{Cu}$ complexes ${ }^{37}$. These results cannot differentiate whether all chains in the film are undergoing oxidation to the same extent, or if parts of the film are left unoxidised. The estimation of $(27 \pm 13.5) \%$ given above should thus be taken as a lower limit for the maximum proportion of copper centres susceptible to oxidation in a single chain.

To further understand the evolution of the electronic structure of metallopolymer 1, we carried out spectroelectrochemistry upon 1 in solution. Changes in optical absorption as the potential was increased are assigned to the oxidation of $\mathrm{Cu}^{\prime}$ to $\mathrm{Cu}^{\prime \prime}$ (Fig. S20). The appearance and growth of a broad, low-energy absorption band at ca. $3000 \mathrm{~nm}$ was observed upon oxidation of 1 . This band was only generated in systems containing multiple copper centres (Fig. S21) and was assigned to IVCT among the metal centres at the core of 1 , consistent with motion of charges in the $d_{z}^{2}$ SOMO between copper centres ${ }^{33,38}$. We also found that longer chains required a higher applied potential to bring about $\mathrm{Cu}^{\prime}$ oxidation than short oligomers, and that this oxidation process was electrochemically irreversible (Fig. S18). We infer that longer helices undergo more structural reorganisation in order to optimise binding of $\mathrm{Cu}^{\mathrm{ll}}$. We hypothesise this reorganisation to involve a winding of the helix, as described below and in Supplementary Information Section 3.11. 


\section{Discussion}

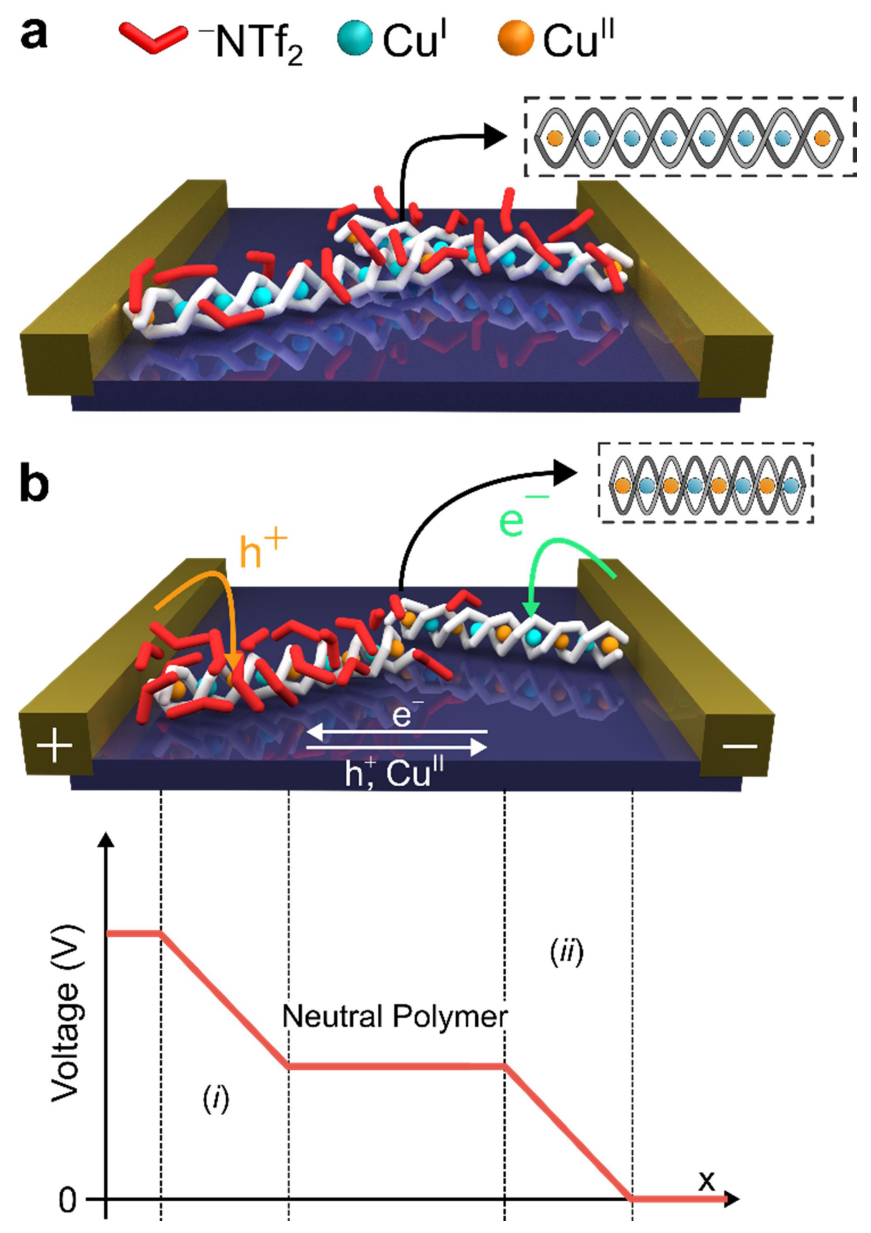

Fig. 4 | Device working mechanism and resistive switching. An idealised schematic representation of metallopolymer 1 in an in-plane device; a real device contains chains with a distribution of orientations in the plane parallel to the substrate, with the majority of them not being in direct contact with the electrodes. a, The metallopolymer in its native state prior to poling, containing $\mathrm{Cu}^{\prime \prime}$ only at the termini of the chain, is positioned between the electrodes. b, (top) Applying a voltage bias across the device triggers ${ }^{-} \mathrm{NTf}_{2}$ migration; the potential drops across the surface-charge-dipole (SCD) regions formed at the electrodes (bottom), promoting oxidation of $\mathrm{Cu}^{\prime}$ to $\mathrm{Cu}^{\prime \prime}$ at the SCD layer close to the positive electrode. Holes are injected into region $(i)$ while electrons are injected into region (ii). ( $\mathrm{NTf}_{2}-$ red, $\mathrm{Cu}^{\prime}$ - cyan, $\mathrm{Cu}^{\prime \prime}$ - orange)

Based on the results presented above, we propose the following mechanism to describe the resistive switching observed in metallopolymer 1 . In its pristine state, 1 contains a core of $\mathrm{Cu}^{\prime}$ atoms with $\mathrm{Cu}^{\prime \prime}$ localised at its termini (Fig. 4a). This $\mathrm{Cu}^{\prime \prime}$ is charge-balanced and stabilised by the presence of radical anionic charge on the ligand strands ${ }^{39,40}$. When a voltage bias is applied, the ${ }^{-} \mathrm{NTf}_{2}$ counteranions of 1 migrate, establishing SCD layers at each electrode (Fig. 4b) that facilitate the injection of holes and electrons from the electrodes into 1. The creation of these SCD layers at the electrodes manifests in the hysteresis observed in Fig.1d. The electric field at the positive electrode is strong enough to oxidise $\mathrm{Cu}^{\prime}$

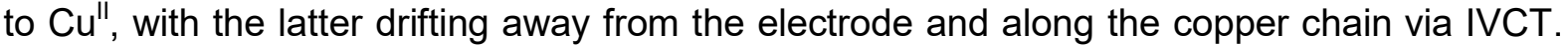
As $\mathrm{Cu}^{\prime \prime}$ migrates away, new $\mathrm{Cu}^{\prime}$ is oxidised until the process becomes energetically unfavourable at the potential applied across the device. We hypothesise that counteranion migration is a required first step to produce the SCD layers that assist oxidation in our 
present devices, where the inter-electrode separation is large, but that such migration would not be necessary in devices with smaller inter-electrode distances.

The increased proportion of $\mathrm{Cu}^{\prime \prime}$ in metallopolymer 1 causes a compression of the double helix ${ }^{32,41}$, whereby a stabilising Jahn-Teller effect acting on the $\mathrm{d}^{9} \mathrm{Cu}^{11}$ lifts the degeneracy of the $d_{z}^{2}$ and $d_{x-y}^{2}{ }^{2}$ orbitals. This process brings about a ground state with $\mathrm{a} \mathrm{dzOMO}_{\mathrm{z}}{ }^{2} \mathrm{SOMO}^{34,35,42}$ and leads the helix to 'wind up' to provide a coordination environment closer to the square planar preference of $\mathrm{Cu}$ ". This effect would shorten $\mathrm{Cu}-\mathrm{Cu}$ distances and improve orbital overlap between adjacent copper centres ${ }^{41}$ through the core. The compression of the helix and the ability of the ligands of 1 to support additional charge ${ }^{39,40}$ thus stabilise the $\mathrm{Cu}^{\text {II }}$ centres that are formed, preventing their re-reduction and giving rise to a long-lived chargeseparated state. To return to the initial higher-resistance state, the activation barrier to helixunwinding must be overcome ${ }^{32}$, which happens more rapidly when the material is heated.

We conclude that the linearly-arrayed $d_{z}^{2}$ orbitals form a partly-filled band that supports charge transport along the $\mathrm{Cu}$ core of 1 . Poled devices thus contain conductive channels consisting of partly oxidised double-helical metallopolymer chains. To further test the role of the copper chain in charge transport, we characterised diodes comprising metallopolymer 1 sandwiched between two hole-injecting contacts (ITO/PEDOT:PSS and $\mathrm{MoO}_{3} / \mathrm{Au}$ ). Since the helical chains lie in the plane of the substrate, these devices probe charge transport in a direction perpendicular to them. At electric fields comparable with those used in the in-plane devices, we observed no hysteresis and no resistive switching in the vertical devices (Supplementary Information Section 3.9). Furthermore, we found that in this configuration holes are transported via inter-chain hopping, with the copper array not being involved in charge transport. These results confirmed that the resistive switching observed in in-plane devices involves a change in charge transport behaviour along the copper array and, importantly, that the organic ligand is effective in shielding the copper arrays from one another.

The inference that charge transport in the poled polymer happens along the oxidised copper core is further supported by comparison with 2, a congener of 1 in which $\mathrm{Ag}^{\mathrm{l}}$ was used in place of $\mathrm{Cu}^{\prime}$. Polymer 2 has the same structure as 1, with helically wrapped ligand strands surrounding a core of $\mathrm{Ag}^{\prime}$ ions (Supplementary Information Section 2). The $\mathrm{d}^{9} \mathrm{Ag}^{\prime \prime}$ state is higher in energy than $\mathrm{d}^{9} \mathrm{Cu}^{\prime \prime}{ }^{43}$. Polymer 2 showed 4-5 orders of magnitude lower conductivity than 1 (Fig. S8) and we concluded that poling produced no $\mathrm{Ag}^{\text {"I }}$ within 2. Finally, DFT calculations on dicopper $(\mathrm{I})$ and disilver $(\mathrm{I})$ model oligomers showed the HOMO residing principally on copper in the former case, but mostly on the organic ligands, and only minimally on silver, in the latter case (Supplementary Information Section 3.12).

As noted above, fully switched devices exhibit bulk conductivities of $2 \mathrm{~S} \mathrm{~m}^{-1}$ (Supplementary Information Section 4.1), but this value may be subject to future optimisation. This conductivity is comparable to values reported for such one-dimensional conductors as Krogmann $^{12}$ and Magnus salts ${ }^{13,16}$, the conductivity of which has been measured on single crystals. Our study, in contrast, probed the bulk behaviour of relatively disordered, solutionprocessed films. In such films, the current flow is impeded by morphological traps and by the end groups of the metallopolymer, which may act as electronic traps. Furthermore and as mentioned above, some of the chains may not have been fully converted into their oxidised, highest-conductivity, state. Single chains or crystals of polymer 1, oriented parallel to the electric field, may ultimately display higher conductivities.

The observation of high electrical conductivity in nominally $\mathrm{Cu}^{\prime}$ materials has previously been associated with disproportionation to $\mathrm{Cu}$ and $\mathrm{Cu}^{\prime \prime}$, whereby external stimuli such as electrical 
bias or high pressure generate conductive filaments of metallic copper ${ }^{44,45}$. We conclude that such irreversible disproportionation is not taking place in 1, however, in light of the reversibility of the voltage-driven increase in electrical conductivity (Fig. 2c). This observation indicates that the polymer chain remains intact upon transition to higher-conductivity states. In contrast, we infer that exposure of sample 1 to air in the low-resistance state results in irreversible oxidation of the negatively charged ligand, leading to sample degradation as the $\mathrm{Cu}^{\prime \prime}$ centres repel each other coulombically (Supplementary Information Section 3.10).

\section{Conclusions}

In this work we have demonstrated that a linear array of copper atoms, constructed by selfassembly of helical metallopolymers, can be made conductive upon oxidation via an externally applied electric field. A large proportion (ca. 25\%) of copper ions in the array is oxidised from $\mathrm{Cu}^{\prime}$ to $\mathrm{Cu}^{\prime \prime}$, a change that is accommodated by a physical "winding" distortion of the helix and by the organic ligand strand taking up anionic charge. The resulting change in electrical conductivity of the polymer can be retained over years, prevented from reversion to the initial state by the distortion of the helix. The organic strands also isolate the molecular wire from other adjacent chains in thin solid films, allowing the properties of isolated atomic chains to be exploited in bulk thin films. These copper helical metallopolymers thus represent an easily accessible platform for the study and application of charge transport at the nanoscale, with access to fine-grained control over the structure-property relations. 


\section{Acknowledgements}

Chris Amey is acknowledged for performing the XPS measurements on thin film samples. The work presented here was funded by the UK Engineering and Physical Sciences Research Council (EPSRC EP/P027067/1, EP/M01083x/1 and EP/M005143/1) and the European Research Council (ERC695009). S.S. and H.S. acknowledge funding from ERC Synergy grant SC2 (no. 610115).

\section{Author Contributions}

J.L.G. synthesised the materials and characterised them by CD, CV, DLS, NMR, UV-Vis and TEM. J.L.G. and D.D.N. performed all the experiments and analysed all the data. E.W.E. and W.K.M. helped with the ESR measurements on frozen solutions. E.W.E. fitted the ESR traces of frozen solutions to extract $g$-values and number of spins, performed the DFT calculations and helped with ultrafast spectroscopy. S.P.S. and J.T.D. fabricated the patterned substrates for in-plane devices. S.P.S. helped with preliminary electrical characterisation of in-plane devices. S.S. fabricated the substrate for in-situ ESR, helped with the measurement and fitted the ESR traces to extract $g$-value and number of spins. A.P. helped with the synthesis of the silver metallopolymer. D.D.N. conceived the project. D.D.N. and J.R.N. supervised the research. J.L.G., D.D.N., R.H.F. and J.R.N. wrote the manuscript, with input from all the authors.

\section{Data availability}

All data that support the findings of this study are included within the Article and its Supplementary Information, and are also available from the authors upon request.

\section{References}

1. Hirschberg, J. H. K. K. et al. Helical self-assembled polymers from cooperative stacking of hydrogen-bonded pairs. Nature 407, 167-170 (2000).

2. Tanaka, K., Tengeiji, A., Kato, T., Toyama, N. \& Shionoya, M. A discrete selfassembled metal array in artificial DNA. Science 299, 1212-1213 (2003).

3. Kang, J. et al. A rational strategy for the realization of chain-growth supramolecular polymerization. Science 347, 646-651 (2015).

4. Wolf, M. O. \& Wrighton, M. S. Tunable Electron Density at a Rhenium Carbonyl Complex Coordinated to the Conducting Polymer Poly[5,5'-(2-thienyl)-2,2'-bithiazole]. Chem. Mater. 6, 1526-1533 (1994).

5. Wolf, M. O. Transition-metal-polythiophene hybrid materials. Adv. Mater. 13, 545-553 (2001).

6. Whittell, G. R., Hager, M. D., Schubert, U. S. \& Manners, I. Functional soft materials from metallopolymers and metallosupramolecular polymers. Nat. Mater. 10, 176-188 (2011).

7. Hu, B. et al. Inorganic-organic hybrid polymer with multiple redox for high-density data storage. Chem. Sci. 5, 3404-3408 (2014).

8. Stanley, J. M. \& Holliday, B. J. Luminescent lanthanide-containing metallopolymers. 
Coord. Chem. Rev. 256, 1520-1530 (2012).

9. Cui, B. Bin et al. Tuning of resistive memory switching in electropolymerized metallopolymeric films. Chem. Sci. 6, 1308-1315 (2015).

10. Ma, Y. et al. Metal complex modified azo polymers for multilevel organic memories. Nanoscale 7, 7659-7664 (2015).

11. Tsai, C. L. et al. Zinc and linkage effects of novel porphyrin-containing polyimides on resistor memory behaviors. RSC Adv. 6, 88531-88537 (2016).

12. Krogmann, K. Planar Complexes Containing Metal-Metal Bonds. Angew. Chemie Int. Ed. English 8, 35-42 (1969).

13. Sakai, K., Ishigami, E., Konno, Y., Kajiwara, T. \& Ito, T. New partially oxidized 1-D platinum chain complexes consisting of carboxylate-bridged cis-diammineplatinum dimer cations. J. Am. Chem. Soc. 124, 12088-12089 (2002).

14. Bera, J. K. \& Dunbar, K. R. Chain compounds based on transition metal backbones: New life for an old topic. Angew. Chemie - Int. Ed. 41, 4453-4457 (2002).

15. Caseri, W. R. et al. '(Hot-)water-proof', semiconducting, platinum-based chain structures: Processing, products, and properties. Adv. Mater. 15, 125-129 (2003).

16. Hendon, C. H. et al. One-dimensional Magnus-type platinum double salts. Nat. Commun. 7, 1-6 (2016).

17. Cotton, F. A., Murillo, C. A. \& Walton, R. A. Multiple Bonds Between Metal Atoms. Multiple Bonds Between Metal Atoms (2005).

18. Berry, J. F. Metal-metal bonds in chains of three or more metal atoms: From homometallic to heterometallic chains. in Structure and Bonding (ed. Parkin, G.) vol. 136 1-28 (Springer, Berlin, 2010).

19. Mashima, K. Linearly aligned metal clusters: Versatile reactivity and bonding nature of tetrametal M-Mo-Mo-M complexes (M = Pt, Pd, Ir, and Rh) supported by 6diphenylphosphino-2-pyridonato ligand. Bull. Chem. Soc. Jpn. 83, 299-312 (2010).

20. Givaja, G., Amo-Ochoa, P., Gómez-García, C. J. \& Zamora, F. Electrical conductive coordination polymers. Chem. Soc. Rev. 41, 115-147 (2012).

21. Uemura, K. One-dimensional complexes extended by unbridged metal-metal bonds based on a HOMO-LUMO interaction at the $\mathrm{d}: \mathrm{Z2}$ orbital between platinum and heterometal atoms. Dalt. Trans. 46, 5474-5492 (2017).

22. Chen, I. W. P. et al. Conductance and stochastic switching of ligand-supported linear chains of metal atoms. Angew. Chemie - Int. Ed. 45, 5814-5818 (2006).

23. Liu, I. P. C. et al. A new generation of metal string complexes: Structure, magnetism, spectroscopy, theoretical analysis, and single molecular conductance of an unusual mixed-valence linear [Ni5]8+ complex. Chem. - A Eur. J. 13, 8667-8677 (2007).

24. Ismayilov, R. H. et al. Two linear undecanickel mixed-valence complexes: Increasing the size and the scope of the electronic properties of nickel metal strings. Angew. Chemie - Int. Ed. 50, 2045-2048 (2011).

25. Greenfield, J. L., Rizzuto, F. J., Goldberga, I. \& Nitschke, J. R. Self-Assembly of Conjugated Metallopolymers with Tunable Length and Controlled Regiochemistry. Angew. Chemie - Int. Ed. 56, 7541-7545 (2017).

26. Greenfield, J. L. et al. Unraveling Mechanisms of Chiral Induction in Double-Helical 
Metallopolymers. J. Am. Chem. Soc. 140, 10344-10353 (2018).

27. Pei, Q., Yu, G., Zhang, C., Yang, Y. \& Heeger, A. J. Polymer Light-Emitting. Science 269, 1086-1088 (1995).

28. Sangwan, V. K. et al. Multi-terminal memtransistors from polycrystalline monolayer molybdenum disulfide. Nature 554, 500-504 (2018).

29. Jeffery, J. C. et al. Localization and delocalization in a mixed-valence dicopper helicate. Inorg. Chem. 46, 2417-2426 (2007).

30. Holland, J. M. et al. Copper complexes of 2,6-bis(iminomethyl)pyridine derivatives and of I,3-bis(pyridin-2-yl)pyrazole. Effects of ligand bulk and conformational strain on the ground state of a six-co-ordinate copper(n) ion. J. Chem. Soc. Dalt. Trans. 3316-3324 (2000) doi:10.1039/b005105p.

31. Roessler, M. M. \& Salvadori, E. Principles and applications of EPR spectroscopy in the chemical sciences. Chem. Soc. Rev. 47, 2534-2553 (2018).

32. Dicke, B. et al. Transferring the entatic-state principle to copper photochemistry. Nat. Chem. 10, 355-362 (2018).

33. D'Alessandro, D. M. \& Keene, F. R. Intervalence charge transfer (IVCT) in trinuclear and tetranuclear complexes of iron, ruthenium, and osmium. Chem. Rev. 106, 22702298 (2006).

34. Hitchman, M. A. The influence of Vibronic Coupling on the Spectroscopic Properties and Stereochemistry of Simple 4- and 6-Coordinate Copper(II) Complexes. Comments Inorg. Chem. 15, 197-254 (1994).

35. Hathaway, B. J. \& Billing, D. E. The electronic properties and stereochemistry of mono-nuclear complexes of the copper(II) ion. Coord. Chem. Rev. 5, 143-207 (1970).

36. Gerson, F. \& Huber, W. Electron Spin Resonance Spectroscopy of Organic Radicals. Electron Spin Resonance Spectroscopy of Organic Radicals (Wiley-VCH Verlag $\mathrm{GmbH} \&$ Co. KGaA, 2003). doi:10.1002/3527601627.

37. Mankad, N. P., Antholine, W. E., Szilagyi, R. K. \& Peters, J. C. Three-coordinate copper(I)amido and aminyl radical complexes. J. Am. Chem. Soc. 131, 3878-3880 (2009).

38. Hua, C. et al. Through-Space Intervalence Charge Transfer as a Mechanism for Charge Delocalization in Metal-Organic Frameworks. J. Am. Chem. Soc. 140, 66226630 (2018).

39. Khusnutdinova, J. R. \& Milstein, D. Metal-Ligand Cooperation. Angew. Chemie - Int. Ed. 54, 12236-12273 (2015).

40. de Bruin, B., Bill, E., Bothe, E., Weyhermüller, T. \& Wieghardt, K. Molecular and Electronic Structures of Bis(pyridine-2,6-diimine)metal Complexes [ML 2 ](PF 6 ) n ( $n$ = 0, 1, 2, 3; M = Mn, Fe, Co, Ni, Cu, Zn) †. Inorg. Chem. 39, 2936-2947 (2000).

41. Schultz, D. et al. Helicate extension as a route to molecular wires. Chem. - A Eur. J. 14, 7180-7185 (2008).

42. Whangbo, M. H. \& Hoffmann, R. The Band Structure of the Tetracyanoplatinate Chain. J. Am. Chem. Soc. 100, 6093-6098 (1978).

43. Grochala, W. \& Mazej, Z. Chemistry of silver ( II ): a cornucopia of peculiarities † Subject Areas : Phil. Trans. R. Soc. A 373, 0-11 (2015). 
44. Gentile, A. L. Electric breakdown mechanism in cuprous chloride single crystals. Appl. Phys. Lett. 9, 237-239 (1966).

45. Kopelevich, Y., Da Silva, R. R. \& Camargo, B. C. Unstable and elusive superconductors. Phys. C Supercond. its Appl. 514, 237-245 (2015). 